Cahiers du MONDE RUSSE

\section{Cahiers du monde russe}

Russie - Empire russe - Union soviétique et États indépendants

42/2-4 | 2001

La police politique en Union soviétique, 1918-1953

\title{
Alliés ou ennemis ? Le GUPVI-NKVD, le Komintern et les « Malgré-nous ». Le destin des prisonniers de guerre français en URSS (1942-1955).
}

Le destin des prisonniers de guerre français en URSS (1942-1955)

\section{Gaël MOULLEC}

\section{OpenEdition}

\section{Journals}

Édition électronique

URL : https://journals.openedition.org/monderusse/8473

DOI : $10.4000 /$ monderusse. 8473

ISSN : 1777-5388

Éditeur

Éditions de l'EHESS

Édition imprimée

Date de publication : 1 avril 2001

Pagination : 667-678

ISBN : 2-7132-1398-3

ISSN : $1252-6576$

Référence électronique

Gaël MOULLEC, «Alliés ou ennemis ? Le GUPVI-NKVD, le Komintern et les « Malgré-nous ». Le destin des prisonniers de guerre français en URSS (1942-1955). ", Cahiers du monde russe [En ligne], 42/2-4 I 2001, mis en ligne le 01 janvier 2007, consulté le 02 septembre 2022. URL : http://

journals.openedition.org/monderusse/8473; DOI : https://doi.org/10.4000/monderusse.8473 
chercher : repérer : avancer

Cet article est disponible en ligne à l'adresse :

http://www.cairn.info/article.php?ID REVUE=CMR\&ID NUMPUBLIE=CMR 422\&ID ARTICLE=CMR 4220667

Alliés ou ennemis ? Le GUPVI-NKVD, le Komintern et les «Malgré-nous ». Le destin des prisonniers de guerre français en URSS (1942-1955). Le destin par Gaël MOULLEC

\section{Editions de l'EHESS | Cahiers du monde russe}

2001/2-3-4 - Vol 42

ISSN 1252-6576 | ISBN 2713213983 | pages 667 à 678

Pour citer cet article :

-MOULLEC G., Alliés ou ennemis ? Le GUPVI-NKVD, le Komintern et les « Malgré-nous ». Le destin des prisonniers de guerre français en URSS (1942-1955). Le destin des prisonniers de guerre français en URSS (1942-1955), Cahiers du monde russe 2001/ 2-3-4, Vol 42, p. 667-678.

Distribution électronique Cairn pour les Editions de l'EHESS.

(C) Editions de l'EHESS. Tous droits réservés pour tous pays.

La reproduction ou représentation de cet article, notamment par photocopie, n'est autorisée que dans les limites des conditions générales d'utilisation du site ou, le cas échéant, des conditions générales de la licence souscrite par votre établissement. Toute autre reproduction ou représentation, en tout ou partie, sous quelque forme et de quelque manière que ce soit, est interdite sauf accord préalable et écrit de l'éditeur, en dehors des cas prévus par la législation en vigueur en France. Il est précisé que son stockage dans une base de données est également interdit. 


\section{ALLIÉS OU ENNEMIS ? LE GUPVI-NKVD, LE KOMINTERN ET LES «MALGRÉ-NOUS »}

\section{Le destin des prisonniers de guerre français en URSS}

$(1942-1955)^{1}$

Pour les Alsaciens-Lorrains, la Seconde Guerre mondiale débute plus tôt que pour les «Français de l'intérieur». Le 1er septembre 1939, alors que les troupes allemandes entrent en Pologne, le gouvernement français donne l'ordre d'évacuer les populations de la zone frontalière. En quelques jours, des milliers d'AlsaciensLorrains quittent tout pour se retrouver sur les routes et dans les gares dans une sorte de répétition générale, l'ordre en plus, du grand exode de 1940. Répartis dans plusieurs régions françaises, 600000 Alsaciens-Lorrains passeront la drôle de guerre loin de chez eux, s'interrogeant perpétuellement sur la date de leur retour au pays. Le 10 mai 1940, les troupes allemandes attaquent par la Belgique. La France s'effondre. À l'Est, les villes de Metz et de Strasbourg résistent respectivement jusqu'au 17 et 19 juin.

Immédiatement après l'entrée des troupes allemandes, les rues et les communes d'Alsace perdent leur nom français et se voient attribuer des toponymes plus

\footnotetext{
1. Le présent article se concentre sur le destin des Malgré-nous, ces Français originaires d'Alsace-Lorraine incorporés de force dans l'armée allemande et dont plusieurs dizaines de milliers seront faits prisonniers par l'Armée rouge. Le cas des militaires français évadés des camps allemands et passés en URSS entre juin 1940 et juin 1941, tout comme le cas des supplétifs français de la LVF (Légion des Volontaires Français contre le Bolchevisme) ne sont pas étudiés ici. On pourra compléter la lecture de cet article par les ouvrage suivants : Pierre Rigoulot, La tragédie des Malgré-nous. Tambov, le camp des Français, Paris, Denoël, 1990 ; Bernard et Gérard Le Marec, L'Alsace dans la guerre 39-45, Roanne, Horvath, 1988 ; Gustav Degen, Malgré-nous de la Wehrmacht à Tambov, Colmar, Alsatia, 1951, et enfin par le numéro spécial de la revue Saisons d'Alsace, 39/40, été-automne 1971.
} 
« germaniques ». Dès le 10 juillet, les préfets et les sous-préfets français en poste en Alsace-Lorraine sont arrêtés et expulsés vers Paris. Durant les premières semaines de l'Occupation, l'Alsace-Lorraine subit une politique de « regermanisation » qui se traduit par la nomination de deux Gauleiters: Robert Wagner en Alsace et Joseph Bürckel en Lorraine. Les deux territoires français perdent leur identité administrative : la Lorraine est intégrée, avec la Sarre et le Palatinat, dans la région de la Westmark, tandis que l'Alsace se trouve réunie au Pays de Bade.

Rapidement, l'usage de la langue française est interdit dans l'administration et les écoles, puis à l'église. Les services administratifs sont intégrés à ceux du Reich (Postes, Chemins de fer, Douanes...) alors que le gouvernement de Vichy reste sans réaction notable. Par un système d'interdiction de retour, de déportation ou d'expulsion vers la France, les Allemands tentent de casser toute résistance à la germanisation forcée en épurant les deux régions des familles qui ne sont pas de « race allemande » ou qui sont jugées trop proches de la France. À la suite des premiers actes de résistance, un camp de « rééducation », réservé aux opposants les plus décidés, est même créé à Schirmeck.

Poursuivant leur politique de germanisation, les autorités d'occupation publient à l'été 1942 des décrets attribuant de droit la nationalité du Reich aux personnes « de souche allemande ». Une telle définition s'appliquait aux personnes ayant au moins deux grand-parents nés en Alsace-Lorraine, au Luxembourg ou en Allemagne. À l'époque, la majorité de la population de ces deux régions correspondait à cette définition. Désormais les Alsaciens-Lorrains pouvaient ainsi être « légalement » enrôlés dans la Wehrmacht.

Le drame des Malgré-nous - ces Français d'Alsace-Lorraine incorporés de force dans la Wehrmacht - débute donc à l'été 1942 quand, pour faire face aux pertes subies sur le front russe depuis juin 1941, les autorités d'occupation instaurent pour les jeunes gens des deux régions françaises un «service obligatoire dans l'armée allemande ».

Toutefois, les officiers allemands ne se font aucune illusion quant à la loyauté de ces nouvelles recrues envers le Reich. Des mesures sont prises pour limiter la proportion des Alsaciens-Lorrains présents dans une même unité, jamais plus de $5 \%$ des effectifs. Il est interdit de les affecter dans les services de reconnaissance, de renseignement, dans l'aviation, ou sur les navires de guerre. Enfin ils ne peuvent servir en France occupée, en Belgique ou au Luxembourg.

Dès les premiers jours de l'incorporation, les actes de résistance et d'insoumission se multiplient. Beaucoup de jeunes sont arrêtés et leur famille déportée au cœur de l'Allemagne. D'autres sont abattus en essayant de fuir par la frontière suisse, ou sont internés au camp de Schirmeck. Enfin certains tentent par tous les moyens - automutilation ou mutilation volontaire médicalisée - d'échapper à la conscription. Au total 130000 Alsaciens-Lorrains des classes 1928 à 1947 sont incorporés de force dans l'armée allemande. Près de $90 \%$ d'entre eux combattront sur le front de l'Est.

Dans la première moitié de 1943, le renseignement militaire soviétique fait état d'un nombre important de désertions chez les Alsaciens-Lorrains. Les transfuges 
sont interrogés et certains de leurs propos rapportés dans des bulletins confidentiels publiés par la VII e section de la Direction politique centrale de l'Armée rouge ${ }^{2}$.

Ainsi Louis Dirheimer, incorporé en octobre 1942, déserte quelques jours après son arrivée sur le front de l'Est en avril 1943 :

« Je suis Lorrain. Mon frère a été tué en France durant les affrontements contre les Allemands. Alors à quoi bon combattre pour les Allemands contre les Russes. L'idée de me rendre m'est venue dès le premier jour de la mobilisation à l'armée allemande [...] D'ailleurs la radio anglaise l'a dit, tous les Lorrains mobilisés de force par Hitler pour faire la guerre contre la Russie doivent passer chez les Russes. Ils quitteront les camps de prisonniers pour l'Angleterre où ils auront la possibilité de s'enrôler dans les troupes de De Gaulle et de Giraud pour combattre l'Allemagne. $»^{3}$

Paul Kele, né a Sarreguemines, se rend en mai 1943 et témoigne du même état d'esprit :

«Personne ne croit ce que racontent les officiers allemands sur les atrocités commises par les Russes. Cependant beaucoup craignent des représailles contre leur famille. On craint également d'être tué par les Allemands si nous tentons de nous rendre. Quand je suis parti pour l'armée, mes parents m'ont dit de me rendre dès que je serai sur le front. Ma mère m'a conseillé d'avoir toujours sur moi un mouchoir blanc et de l'employer comme signal pour me rendre. Avec mes camarades, nous avions aussi discuté de l'éventualité de passer chez les Russes à la première occasion. Avec le soldat Philippe Krez, nous avions même convenu de passer chez les Russes ensemble ; mais sur le front nous avons été mis dans des unités différentes. »

Mobilisé en janvier 1943, Aloïs Eger se rend en mai 1943 :

«Les Alsaciens sont contre Hitler, ils veulent se rendre aux Russes pour gagner l'armée de De Gaulle. Maintenant il est impossible de passer car nous sommes très surveillés par nos officiers. Les Alsaciens espèrent que les Russes déclencheront rapidement l'offensive car ainsi, cachés dans une tranchée, nous pourrons nous rendre avec moins de risques. »

Passés à l'Armée rouge en juin 1943, Albert Heinel et Marcel Schmidt rappellent que « seul un nombre infime d'Alsaciens a un état d'esprit conciliateur, 99,5\% sont contre les hitlériens, tandis que seuls $0,5 \%$ sont pour la collaboration avec les Allemands ».

Tous témoignent de leur attachement à la France et nombre d'entre eux se rendent aux Soviétiques dans l'espoir de rejoindre les troupes de la France libre.

2. VII otdel glavnogo političeskogo upravlenija raboče-krestjan'skoj Krasnoj Armii.

3. Bulletin d'information de la VII' section de la Direction politique centrale de l'Armée rouge, 9 août 1943, Rossijskij Gosudarstvennyj Arhiv Social'no-Političeskoj Istorii (RGASPI), 517/1/ 1947/50-56. 
Influencés par la propagande gaulliste, ils n'imaginent jamais que leur route vers l'Angleterre se terminera dans les camps de prisonniers de guerre dépendants de la Direction centrale des prisonniers de guerre et des internés du commissariat du peuple aux Affaires intérieures (Glavnoe upravlenie NKVD SSSR po delam voennoplennyh $\mathrm{i}$ internirovannyh -GUPVI-NKVD $)^{4}$.

La création du GUPVI-NKVD remonte à l'invasion de la Pologne orientale par les troupes soviétiques. Par un décret du 19 septembre 1939, L. P. Berija, commissaire du peuple aux Affaires intérieures, met en place les premiers camps de prisonniers. Cette nouvelle organisation est intégrée au commissariat du peuple à l'Intérieur au niveau de l'Union comme dans les républiques et les régions pour les questions liées au contre-espionnage et à la logistique (nourriture, soins médicaux).

Dans un premier temps, la tâche du GUPVI est de prendre en charge les prisonniers dans des camps temporaires, proches du front, et de les transférer dans les camps d'internement définitif, puis de les enregistrer et de les mettre au travail le plus rapidement possible. Tout change avec l'attaque allemande contre l'Union soviétique. Immédiatement, 30 nouveaux camps sont créés dans la zone s'étendant entre le front et la Russie centrale et plus particulièrement en Ukraine et en Carélie. Un mois après le début des hostilités, suite à l'avancée allemande, seuls 19 camps restaient en activité. Ainsi durant les premiers mois du conflit, le GUPVI n'a qu'une existence virtuelle, ses principales fonctions sont remplies par les structures du GULag pour la logistique, et par les structures de la Seconde direction du NKVD pour le contre-espionnage. À l'époque, l'administration centrale du GUPVI ne compte que 39 personnes.

Nouveau remaniement à partir de décembre 1941 et de l'arrêt de l'offensive allemande sur Moscou : le 27 décembre 1941, le GOKO (Gosudarstvennyj komitet oborony - Comité d'État à la défense) adopte un décret autorisant le GUPVI à prendre en charge 26 nouveaux camps pour faire face à l'afflux de nouveaux prisonniers. Les revers de l'armée allemande à la suite de la bataille de Stalingrad conduisent à l'ouverture de nouveaux camps et à la mise en place d'hôpitaux spéciaux pour traiter les prisonniers les plus gravement touchés.

À la fin de 1943, le GUPVI compte déjà plus d'une cinquantaine de camps. Un an plus tard, il contrôle 156 camps d'une capacité totale de 905000 personnes. Durant les derniers mois du conflit, il doit faire face à un afflux toujours plus massif de prisonniers et déploie plus de 102 nouveaux camps de réception et de filtrage dans les arrières immédiats du front. Au total près de 4000 points (camps fixes et itinérants, hôpitaux, bataillons de travail) dépendant du GUPVI recevront près de

\footnotetext{
4. Les travaux les plus complets sur cette administration soviétique ont été menés par le chercheur autrichien Stefan Karner. Voir en particulier : S. Karner, Im Archipel GUPVI, Kriegsgefangenschaft und Internierung in der Sowjetunion 1941-1956, Vienne-Munich, 1995 (Kriegsfolgen-Forschung, Bd I) ; Id., « Die sowjetische Hauptverwaltung für Kriegsgefangene und Internierte. Ein Zwischenbericht », Vierteljahreshefte für Zeitgeschichte, 3, 1994 ; Id., «Deutsche Kriegsgefangene und Internierte in der Sowjetunion, 1941-1956», in Problemy voennogo plena. Istorija i sovremenost', Vologda, 1997. La meilleure somme documentaire sur cette Direction centrale est le volume publié par M. M. Zagorul'ko, Voennoplennye v SSSR 1939-1956. Dokumenty i materialy, Moscou, Logos, 2000, 1120 p.
} 
3500000 prisonniers de guerre entre 1941 et 1945, dont un peu plus de 20000 Français. Dans leur cas, le Komintern eut une influence presque égale à celle du GUPVI sur leur destin.

André Marty tient une place prépondérante dans le destin des « Malgré-nous ». Il est chargé, dans le cadre de ses activités au sein du Komintern, de la surveillance des Français présents en URSS. Mutin de la Mer Noire en 1919, communiste de la première heure, dirigeant des Brigades internationales en Espagne, Marty n'en est pas moins un Français, formé sur les bancs de la communale, pour qui les Alsaciens-Lorrains - même revêtus de l'uniforme allemand - sont des compatriotes et doivent, si possible, être traités comme tels.

Début 1943, André Marty tente de recueillir, par l'intermédiaire de plusieurs responsables du Komintern et d'officiers du NKVD, les quelques bribes d'informations disponibles sur les « Français incorporés de force dans l'armée allemande ». Le 19 avril 1943, une responsable d'Inoradio, station soviétique émettant vers les pays occupés, lui annonce que plusieurs Alsaciens-Lorrains ont déserté l'armée allemande et se sont rendus à l'Armée rouge sur le front de Leningrad. Le 6 mai, Walter Ulbricht ${ }^{5}$, lui aussi présent à Moscou, l'informe qu'il a relevé la présence de quatre Français dans les listes de prisonniers qui lui ont été transmises. Le 16 mai, Marty note la publication dans le bulletin d'information de la VII e section de la Direction politique centrale de l'Armée rouge de l'interrogatoire d'un transfuge français, l'Alsacien André Imbs, qui s'est rendu le 10 avril aux troupes soviétiques. Toujours en mai 1943, Marty attire l'attention du général Petrov, responsable du GUPVI-NKVD, sur le sort de trois Alsaciens récemment capturés 6 .

Fort de ces informations, André Marty adresse le 3 juin 1943 un rapport au Bulgare Georgij Dimitrov, secrétaire général du Komintern7, lui demandant d'intercéder auprès des autorités soviétiques pour que les prisonniers de guerre français, à l'exception des membres de la LVF, puissent être réunis dans un même camp de prisonniers car « il ne serait pas juste de les considérer comme des ennemis de l'Union soviétique $»^{8}$. Mais au-delà de l'attribution d'un régime de détention particulier - le terme de privilégié serait ici déplacé - cette séparation des Français du reste des prisonniers de la Wehrmacht devait permettre, dans l'esprit de Marty, une sélection politique de ces hommes en vue de leur utilisation ultérieure par le «PCF ou en tout cas par les forces militaires françaises $»^{9}$. Conséquence immédiate de cette note, le 10 juillet 1943, la Pravda publie sous le titre «La voix de la France » un article d'Ilya Ehrenbourg, écrivain et fidèle propagandiste du régime

5. Walter Ulbricht (1893-1973), membre de l'Union spartakiste, Kominternien, premier secrétaire du PC d'Allemagne de l'Est de 1950 à 1971.

6. RGASPI, 517/1/1947/12

7. Bien que le Komintern ait été officiellement dissous en mai 1943, sa structure administrative reste en place et continue de coordonner l'action des partis communistes européens jusqu'à la fin de la guerre.

8. RGASPI, 517/1/1947/16

9. Ibid. 
soviétique, retraçant l'équipée d'un déserteur alsacien de la Wehrmacht. Pour la première fois le régime soviétique reconnaissait officiellement la singularité du cas des « Malgré-nous ».

Bien qu'il soit impossible de reconstruire le cheminement du rapport de Marty dans le dédale de la bureaucratie du Komintern et de l'administration du GUPVINKVD, son influence directe est indéniable dans la création d'un centre de rassemblement des prisonniers français : le camp $n^{\circ} 188$, situé près du village de Rada, dans la région de Tambov. Ouvert en 1942, ce camp de prisonniers ne comptait que trois Français en juin $1943^{10}$, le mois où Marty rédige son rapport. Une fois prise la décision de rassembler les prisonniers français dans ce camp, les effectifs augmentent rapidement : 42 en août $1943^{11}, 394$ en octobre ${ }^{12}$ et 412 en novembre de la même année ${ }^{13}$. En janvier 1944, le camp de Tambov compte 1039 Français sur les 2751 prisonniers $^{14}$ de plus de 15 nationalités différentes qui y sont internés. Les effectifs du camp culminent au début du mois de septembre 1945 avec près de 11000 prisonniers français.

Capturés sur le front ou passés aux lignes soviétiques, les prisonniers étaient immédiatement soumis à un interrogatoire. Les transfuges se voyaient remettre un « certificat», témoignant de leur désertion, les autres, au gré des circonstances, étaient insultés, voire frappés plus ou moins violemment. Une fois en captivité le sort des uns et des autres ne fut guère différent. Les certificats établis au front furent repris par la suite et ils perdirent toute valeur pour ceux qui avaient réussi à les garder sur eux.

Avant tout, il importe de souligner que, pour rejoindre Tambov, nombre de captifs passaient d'abord dans plusieurs autres camps de transit, souvent plus meurtriers que Tambov même. Au total la présence d'Alsaciens-Lorrains a été attestée, grâce à des documents soviétiques de l'époque, dans plus d'une centaines de camps du GUPVI répartis sur l'ensemble du territoire de l'URSS ${ }^{15}$.

Au gré des diverses fouilles, les prisonniers perdaient le peu d'effets personnels qui étaient encore en leur possession : photos, montres, bottes. Une fois arrivés à destination, ils étaient mis en quarantaine, soignés, puis répartis dans des baraques. Outre la faim, c'est le souvenir de ces baraques, enfoncées de deux à trois mètres sous la terre de sorte que seules les lucarnes des toits laissaient pénétrer l'air et la lumière, qui a marqué le plus profondément les prisonniers de Tambov.

10. Les chiffres cités sont issus des rapports politiques (Politdonesenija) mensuels rédigés par la direction du camp à l'intention de l'administration centrale du GUPVI, Centr Hranenija Istoriko-Dokumental'nyh Kollekcii (CHIDK), 52/5/3/86.

11. CHIDK, 52/5/3/112.

12. CHIDK, 52/5/3/139.

13. CHIDK, $52 / 5 / 3 / 152$

14. CHIDK, 52/5/11/5.

15. Pour plus de détails voir : G. Moullec, Les Prisonniers de guerre français en URSS, Catalogue des Fonds russes, Paris, 1998, 322 p. Rapport remis au Secrétariat d'État aux Anciens combattants et aux victimes de guerre. 
Au quotidien, la vie dans le camp était rythmée par les périodes de travail, un maigre repas et l'éducation politique. Au-delà des activités liées à l'entretien normal du camp, des gardiens et des prisonniers, les Malgré-nous étaient astreints à des travaux physiques particulièrement durs : coupe et transport du bois dans les forêts entourant le camp, construction d'une écluse, extraction de la tourbe. Ces travaux, exécutés quelles que fussent les conditions climatiques, étaient d'autant plus exténuants que l'organisme des prisonniers se trouvait affaibli par la maladie et la médiocrité des rations alimentaires.

Les rapports sanitaires établis par les autorités françaises au retour des prisonniers sont accablants. Un quart des détenus étaient atteints du typhus, $15 \%$ de la tuberculose. La dysenterie infectieuse, la pneumonie et la pleurésie firent aussi des ravages. Enfin, tous les prisonniers accusaient d'importantes pertes de poids. À leur retour, le poids moyen des prisonniers, calculé sur un échantillon de 1400 personnes, était de 42 kilos.

Selon les témoignages des anciens de Tambov, « la ration journalière comprenait 600 grammes de pain noir, trois soupes, une portion de bouillie de céréales » pour les prisonniers de la première catégorie, physiquement aptes au travail. Ainsi de l'été 1944 à l'été 1945, chaque détenu ne recevait que l'équivalent de 1340 calories par jour ${ }^{16}$. Des rations «améliorées » étaient servies aux malades, mais les prisonniers mis au cachot, souvent pour une faute bénigne, ne recevaient que 400 grammes de pain et une demi-portion de soupe.

La lecture publique de la presse tenait une place importante dans l'emploi du temps. Réunis dans leur totalité sur la place centrale du camp ou, plus souvent, par groupes dans le «club » réservé à l'éducation politique, les prisonniers prenaient connaissance des discours des dirigeants soviétiques ou des articles des journaux russes concernant la situation en France. Leurs réactions faisaient immédiatement l'objet d'un rapport de la part des responsables politiques du camp. Ainsi en octobre 1943, l'un de ces rapports note que « l'annonce de la libération de la Corse a été accueillie par une ovation des prisonniers [...] de Gaulle est particulièrement populaire parmi eux [...] tous portent sur la poitrine un petit morceau de ruban tricolore. $\gg^{17}$

Les principes de sélection politique prônés par Marty, mais en fait issus de la guerre civile russe où Blancs comme Rouges tentaient de recruter dans leurs rangs les soldats étrangers faits prisonniers, correspondent parfaitement à la vision soviétique et seront appliqués aux prisonniers de guerre de toutes nationalités.

16. La norme officielle adoptée par le GUVPI-NKVD en octobre 1944 (Položenie o voennoplennyh) prévoyait pour les prisonniers aptes au travail la ration journalière suivante : pain de seigle : 600 grammes, céréales : 70 g., viande : 30 g., poisson : 50 g., lard : 10 g., sucre : 17 g., pommes de terre : 400 g., choux : 200 g. (Gosudarstvennyj Arhiv Rossijskoj Federacii-GARF, R-9401/205/12/21-23). Soit un total journalier de plus de 2400 calories. Une telle différence entre la norme et la ration réellement servie aux prisonniers indique l'ampleur des détournements.

17. CHIDK, $52 / 5 / 3 / 139 \mathrm{v}$ 
En novembre 1943, le chef du camp de Tambov rédige un « rapport politique » attirant l'attention de sa hiérarchie sur la qualité de la formation idéologique à laquelle sont soumis les Français. «[...] Par exemple, parmi les prisonniers de guerre français, nous comptons un groupe antifasciste fort de 26 activistes ${ }^{18}$. Il revient une nouvelle fois sur cette question en janvier 1944 pour noter qu'au total le camp compte 600 antifascistes, dont 31 activistes, plus de 40 prisonniers ayant déjà été dirigés vers « l'école des activistes » ${ }^{19}$ au cours des mois précédents. L'idée de créer une telle école revient à G. Dimitrov. Durant la Seconde guerre mondiale, le rôle du Komintern, outre la coordination de l'action des partis communistes des pays occupés, est de former les étrangers présents en URSS, y compris les prisonniers de guerre, afin d'utiliser les plus compétents pour mener des opérations de renseignement derrière les lignes allemandes.

En juillet 1942, Dimitrov note ainsi dans son journal :

«École du temps de guerre. Notre mission, compte tenu de ce qui va se passer après le conflit, est de préparer, dans les délais les plus courts, des fonctionnaires pour les partis et les organisations de jeunesse à l'étranger. Dans cet enseignement, l'étude des événements journaliers de la Guerre, des relations internationales et de la situation politique particulière au pays du stagiaire doit tenir une place centrale. Les cours doivent se construire autour de l'étude des principes marxistes-léninistes, de la formation militaire et en particulier de la lutte clandestine et du soulèvement armé. Un entraînement physique approprié, une bonne conscience politique et morale devront permettre [au stagiaire] de combattre dans les dures conditions de la clandestinité [...]. »20

Peu à peu une nouvelle hiérarchie s'instaure dans le camp de Tambov ; basée sur un conformisme idéologique apparent, elle prend la place de la hiérarchie basée sur le grade. L'encadrement des prisonniers est transféré à ces «antifascistes » qui deviennent chefs de baraquement ou responsables du contrôle des cuisines. Les témoignages réunis par Pierre Rigoulot auprès des anciens de Tambov sont sans appel : «Les Russes leur ont laissé toute liberté pour nous emmerder. La journée, ils étaient dans les cuisines pour manger dans les marmites. L'un d'eux se vantait même d'avaler cinq rations de soupe. Ils étaient tous bien en forme. »

Plus grave encore, ces «antifascistes » en charge de la discipline interne du camp infligent aux prisonniers, au travers d'une «police» principalement composée de Français, des sanctions outrancières pour toute infraction, réelle ou supposée, au règlement. Un ancien de Tambov témoigne :

\section{CHIDK, 52/5/3/153v.}

19. L'école de Krasnogorsk, située aux environs de Moscou, devait former, sous la responsabilité commune du Komintern et des services de renseignement soviétiques, des propagandistes professionnels. Cette formation constituait un préalable indispensable avant que ces activistes ne soient - pour les meilleurs - définitivement recrutés par les services soviétiques pour mener des actions de renseignement sur le territoire français (en France occupée ou en Algérie).

20. G. Dimitrov, Dnevnik, Sofia, Sv. Kliment Okhdinski, 1997, p. 124. 
«Après avoir peiné toute la journée à décharger de la farine à la gare et n'en étant revenu que très tard dans la nuit, je refusais de travailler la journée suivante. Cela me valut huit jours de corvée de w.-c. Alors que la louche à purin était tombée dans la fosse, le chef de la police [...] exigea de moi que je me déshabille pour chercher l'instrument noyé dans cette fosse de trois mètres de profondeur. Ayant refusé, je fus puni de quatorze jours de corvée supplémentaires. $\gg^{21}$

En fait, un véritable système de passe-droit, de marché noir et de combines avait été mis en place pour le seul profit de quelques petits chefs et de leurs sbires. Le tout sous l'œil désabusé des responsables soviétiques du camp, trop contents de « faire du chiffre » en communiquant à leur hiérarchie d'impressionnantes listes nominatives de « sympathisants ».

Croyant servir la France en se rendant à l'Armée rouge, les Malgré-nous se retrouvent confrontés à la dure réalité des camps soviétiques. Les mois passant, ils perdent tout espoir de pouvoir rejoindre les troupes du général de Gaulle. De cette période naît le sentiment - encore vivace aujourd'hui chez certains anciens de Tambov - d'avoir été oubliés par la France. La réalité est toutefois plus complexe.

Dès la fin 1943, ces prisonniers français deviennent des pions dans le jeu d'une diplomatie qui prépare déjà l'après-guerre. Un rapport de Berija, chef du NKVD, adressé à Stalin le 8 janvier 1944 montre comment les dirigeants soviétiques hésitent quant à la politique à suivre envers les Malgré-nous ${ }^{22}$.

Deux solutions sont proposées. La première est de former au sein de l'Armée rouge une brigade «Alsace-Lorraine », utile dans le cas (envisageable avant le 6 juin 1944) où les troupes soviétiques parviendraient jusqu'en France. Plus simplement, la seconde solution prévoyait la remise des prisonniers aux autorités gaullistes pour les engager dans les combats d'Italie.

Le débarquement du 6 juin 1944 met définitivement fin aux hésitations soviétiques et, un mois plus tard, un premier convoi de 1500 hommes quitte le camp de Tambov pour rejoindre l'Afrique du Nord après un long périple.

Ces quelques centaines d'élus, conscients de leur chance ou plus certainement soucieux de ne pas mécontenter les cadres politiques du camp, tout-puissants dans l'établissement de la liste des prisonniers libérables, adoptent, à l'unanimité, une dernière motion pleine d'un enthousiasme appris : « [...] et nous quittons l'URSS aux cris de: "Vive l'amitié franco-soviétique, garantie de la paix européenne. Vivent le peuple soviétique, sa glorieuse Armée rouge, son Grand Chef, le Généralissime Stalin". $»^{23}$

Cependant, en dépit d'une telle bonne volonté, ces 1500 prisonniers français seront les seuls à être libérés avant la capitulation allemande. Avec la fin prévisible du second conflit mondial, ces prisonniers deviennent une monnaie d'échange permettant à tout moment aux Soviétiques de faire pression sur le gouvernement

21. P. Rigoulot, op. cit., p. 24

22. GARF, R-9401/2/64/34.

23. CHIDK, $52 / 4 \mathrm{~m} / 27 / 3$. 
français. De leur côté, soucieuses de ne pas compromettre les relations avec l'URSS, les autorités françaises - du général de Gaulle aux ministres communistes - ne feront jamais du sort des Malgré-nous une question de principe ${ }^{24}$.

Cependant il serait faux de dire que rien n'a été tenté. À de nombreuses reprises, le général Keller, responsable de la mission française de rapatriement présente à Moscou, interroge les Soviétiques sur le destin des Alsaciens-Lorrains - y compris ceux qui servaient dans les troupes $\mathrm{SS}^{25}$-, localisés par ses services. Ces demandes, traitées au cas par cas par les fonctionnaires du GUPVI-NKVD, aboutissent parfois à un rapatriement anticipé des prisonniers concernés.

À l'été 1945, l'Ambassade de France à Moscou adresse une note officielle au ministère soviétique des Affaires étrangères critiquant le «mauvais état de santé des prisonniers arrivant d'URSS ». L'affaire remonte jusqu'à Berija qui couvre immédiatement ses services en adressant à Stalin et à Vyšinskij un rapport détaillé, mais bien loin de la réalité décrite par les survivants.

« Le camp de Tambov [...] est constitué de baraquements confortables réservés aux prisonniers français. Le chef de la mission militaire française, le général Petit, a, lui-même, remercié le Service des relations extérieures du commissariat du peuple à la Défense à la suite de la visite de juillet 1944 durant laquelle il a pu prendre connaissance des conditions de vie des Français. Toutefois, en dépit de bonnes conditions de détention des prisonniers dans les camps du NKVD, l'état physique d'un grand nombre de Français est dégradé, en particulier de ceux qui ont été fait prisonniers dans les dernières semaines de la guerre. $»^{26}$

Plus largement, l'attention, même limitée, que la France porte à l'époque au destin des Malgré-nous donne des résultats, comme le prouvent des documents soviétiques récemment déclassifiés. En septembre 1945, sur les 15139 prisonniers de guerre français recensés à cette date par les autorités soviétiques, 12146 ont déjà quitté l'Union soviétique en direction de la France ${ }^{27}$.

En décembre 1945, le gouvernement soviétique décide de libérer la plus grande partie des prisonniers de guerre, à l'exception de ceux des puissances vaincues (Allemagne, Italie, Japon), toujours internés dans les camps. Les Français encore détenus en URSS, y compris ceux qui avaient servi dans les troupes SS ou les services de renseignements du Reich ${ }^{28}$, sont peu à peu libérés et convoyés vers le camp 186, près d'Odessa, en vue de leur embarquement pour la France. Les années

24. Le livre coordonné par Catherine Klein-Gousseff, Retours d'URSS. Les prisonniers de guerre français et les internés français dans les archives soviétiques, 1945-1951 (Paris, CNRS Éditions, 2001, 428 p.) donne, en dépit du fait que les documents utilisés ne proviennent que d'un seul centre d'archives, un bon éclairage des tractations diplomatiques entre la France et l'Union soviétique.

25. GARF, R-9526/1/207/131

26. GARF, R-9401/2/99/181-182.

27. GARF, R-9401/2/99/182.

28. Directive $n^{\circ} 15$ du NKVD en date du 8 janvier 1946 in Velikaja Otečestvennaja, T. 13 : Inostrannye voennoplennye Vtoroj mirovoj vojny v SSSR, Moscou, Terra, 1996, p. 281. 
passant, le flux des retours diminue, 19 Alsaciens-Lorrains rentrent en France en 1948, 12 en 1949, 1 seul en 1950, 18 en 1951, 4 en 1952, 7 en 1953, aucun en 1954. En 1955, seul Jean-Jacques Remetter revient en France, il sera officiellement le dernier Malgré-nous à être libéré des camps soviétiques.

Les fonds du GUPVI-NKVD, conservés aux Archives spéciales de Moscou, nous donnent une première approximation du nombre total des prisonniers français passés par les camps soviétiques. Toutefois, loin de rendre compte du nombre de prisonniers tombés au mains de l'Armée rouge, les chiffres présentés ici ne reflètent que le nombre de prisonniers arrivés vivants dans un camp permanent d'internement, souvent plusieurs mois après leur capture sur le front.

En effet, bien que les premiers textes réglementaires soviétiques sur les « conditions de détention et de comptage des prisonniers de guerre » aient été promulgués dès août $1941^{29}$, il faudra attendre novembre 1944 pour que des mesures soient prises afin de «mettre en place un comptage individuel des prisonniers dès leur arrivée dans un camp d'internement. $»^{30}$

Ainsi selon les documents disponibles aux Archives spéciales de Moscou (dossiers personnels et cartes individuelles d'enregistrement), 23136 prisonniers de guerre français ont été comptabilisés, 1325 sont morts en captivité et 21811 ont été remis aux autorités de leur pays d'origine. Ces chiffres doivent donc être maniés avec précaution, mais peuvent toutefois être comparés avec ceux des prisonniers d'autres nationalités dont le comptage a été effectué dans conditions identiques.

Avec un taux de perte dans les camps de l'ordre de 5,7\% les Français - tout comme les Hollandais $(4,2 \%)$, les Luxembourgeois $(5,6)$, les Yougoslaves $(6,2 \%)$, les Danois $(7,7 \%)$ et les Belges $(8,8 \%)$ - apparaissent comme ayant été les prisonniers les moins maltraités. Le taux des pertes allemandes s'élève à $15 \%$ et reflète la moyenne générale. Enfin, les Roumains et surtout les Italiens (avec des taux de perte de $29,1 \%$ et de $56 \%$ respectivement) semblent avoir été soumis à des conditions de captivité particulièrement dures.

Plus rapidement libérés que les autres prisonniers, la grande majorité des Malgré-nous ne sera pas confrontée aux conditions d'internement des années 19461947 quand, face à la crise alimentaire qui touche l'ensemble du pays, la direction du GUPVI décide de diminuer encore les rations alimentaires ${ }^{31}$ et d'interrompre la distribution de repas chauds dans les camps de prisonniers ${ }^{32}$.

Toutefois, plus de cinquante ans après la fin de la guerre, le sort de 11000 Alsaciens-Lorrains (20 000 selon d'autres estimations) reste inconnu. Ont-ils été tués au front lors des derniers mois du conflit sans que leur mort ait été enregistrée par une armée allemande en déroute ? Ont-ils été exécutés alors qu'il se rendaient à des

29. Directive $n^{\circ} 1067$ du NKVD en date du 7 août 1941. GARF, R-9041/12/205-12/313-321.

30. Directive $n^{\circ} 254$ du NKVD en date du 16 novembre 1944. GARF, R-9401/12/205-12/112$112 \mathrm{v}$.

31. Directive $n^{\circ} 244$ du MVD en date du 1er octobre 1946. GARF, R-9401/12/205-12/389.

32. Télégramme ${ }^{\circ} 274$ du MVD en date du 14 octobre 1946. GARF, R-9401/12/205-17/256256v. 
troupes soviétiques encore sous le choc des atrocités commises contre les civils russes par d'autres Français, ceux de la LVF ? Plus sûrement, on peut estimer qu'ils ont disparu lors des interminables transferts d'un camp de transit à l'autre, entre leur capture sur le front et leur enregistrement dans un camp permanent d'internement.

Il n'est pas exclu qu'une part minime, mais non négligeable, de ces « disparus » ait en fait été jugée sous l'accusation, réelle ou imaginaire, de crime de guerre et soit décédée dans les camps d'internement bien après la fin des hostilités.

En fin de compte, les incertitudes quant au nombre et au destin de ces 11000 prisonniers de guerre français disparus ne pourront être levées que par une étude approfondie, en Russie, des archives du fameux SMERŠ (Smert' špionam - Mort aux espions) - le renseignement militaire étant la seule administration soviétique à comptabiliser les prisonniers immédiatement après leur capture - et des archives centrales du ministère de l'Intérieur qui conservent les dossiers personnels des prisonniers de guerre étrangers condamnés par des tribunaux soviétiques pour crime de guerre. Mais une telle recherche pourrait soulever bien des questions. En effet, en juillet 1955, au moment où officiellement le dernier Malgré-nous rentre en France, un document du secrétariat du MVD adressé au Comité central du parti atteste que 28 prisonniers de guerre français sont encore détenus dans les camps soviétiques ${ }^{33}$.

Fondation Robert Schuman

29, boulevard Raspail

75007 Paris

e-mail :g.moullec@robert-schuman.org

33. GARF, R-9401/2/465/167a/. Ce document est issu d'un dossier concernant plus largement le rapatriement des prisonniers de guerre allemands et japonais encore présents en URSS en juin 1955 